\title{
Influencia de escenarios cambiantes de estrés hídrico y encharcamiento en la susceptibilidad de Castanea sativa a Phytophthora cinnamomi
}

\author{
Martín M.A. ${ }^{1}$, Vázquez A. ${ }^{1}$, Martín L.M. ${ }^{2}$, Solla A. ${ }^{*}$ \\ ${ }^{1}$ Ingeniería Forestal y del Medio Natural. Universidad de Extremadura. \\ Avenida Virgen del Puerto, 2, 10600 Plasencia, España \\ ${ }^{2}$ Departamento de Genética, ETSIAM, Campus de Rabanales, Universidad de Córdoba, 14071, Córdoba, España. \\ "Autor para correspondencia: asolla@unex.es
}

\begin{abstract}
Resumen
El castaño (Castanea sativa Mill.) es una especie multipropósito de gran importancia económica y cultural en Europa. Por la versatilidad que presenta su aprovechamiento se considera un buen ejemplo de integración entre la distribución de la biodiversidad natural y la generada por el hombre, y un buen modelo para el desarrollo de estrategias encaminadas a combinar conservación y utilización sostenible. Desde hace tiempo, sin embargo, los castañares experimentan una creciente mortalidad debido a la presencia de Phytophthora, probablemente agravada por determinadas circunstancias actuales del cambio climático. El objetivo del trabajo fue cuantificar la influencia de escenarios cambiantes de estrés hídrico $v s$ encharcamiento en la susceptibilidad de C. sativa a P. cinnamomi. Se utilizaron brinzales de una savia germinados a partir de castaña procedente de Hervás (Cáceres). Durante el período vegetativo, la planta fue dividida en tres grupos y sometida a los tratamientos estrés hídrico, condiciones elevadas de hidromorfía, y riego moderado (control). A las seis semanas de tratamientos las plantas fueron inoculadas artificialmente con P. cinnamomi, y a las ocho semanas, sometidas a condiciones cruzadas de estrés hídrico, encharcamiento y riego moderado, de modo que se generaron nueve tratamientos (escenarios cambiantes) y tres controles (escenarios estables). Para constatar la efectividad de los tratamientos se realizaron mediciones periódicas de la humedad del suelo y del estado fisiológico de las plantas. Se presentan resultados de mortalidad y fisiología de las plantas analizadas, y se discuten cuáles son los escenarios más favorables y desfavorables para el castaño, previos y posteriores a la infección por P. cinnamomi.
\end{abstract}

Palabras clave: cambio climático, oomiceto, organismo invasor, tinta del castaño. 


\section{Introducción}

En los sistemas forestales, el cambio global implica severas alteraciones y amenazas generadas principalmente por el cambio climático y el comercio global. En relación al cambio climático, se prevé un aumento de la temperatura global media (Stocker et al., 2013) lo que favorecerá la incidencia de importantes enfermedades en el ámbito forestal (Sturrock et al., 2011; Pautasso et al., 2012; Helfer, 2013; MartínGarcía et al., 2014). También se espera una mayor irregularidad de las precipitaciones, con episodios acusados de estrés hídrico y condiciones prolongadas de encharcamiento (Bailey-Serres y Colmer, 2014), lo que generará nuevas interacciones patógeno-huésped mediadas por dichas situaciones cambiantes (Yáñez-López et al., 2012; Corcobado et al., 2014). Por su parte, el comercio global ha supuesto la aparición de múltiples enfermedades causadas por organismos exóticos, dentro de los cuales destaca el género Phytophthora por su elevado impacto en casi todas las masas forestales (Santini et al., 2013; Jung et al., 2016).

Todo esto hace que, en la actualidad, poder predecir los efectos del cambio global sobre comunidades forestales diezmadas por patógenos invasores sea todo un reto para la comunidad científica (Alberto et al., 2013; Pautasso et al., 2015).

El castaño (Castanea sativa Mill.) es una de las especies multipropósito de mayor importancia económica y cultural en Europa. En España es una especie naturalizada que se utiliza para producción de fruto y madera en comarcas con un gran interés medioambiental, y cuya gestión se hace por métodos tradicionales. Sin embargo, durante las últimas décadas, la gestión del castaño en España y la decadencia de su cultivo han estado asociados estrechamente a un empeoramiento general de su estado sanitario debido a dos enfermedades de origen exótico, la 'tinta' y el 'chancro'. Estas patologías están generadas por el oomyceto Phytophthora cinnamomi Rands y el ascomiceto Cryphonectria parasitica (Murril) ME Barr (=Endothia parasitica), y su introducción en España data de los años 1726 y 1940, respectivamente (Vieitez et al., 1996; Mansilla et al., 2000).

El intercambio de material vegetal y suelo por parte del hombre (Jung et al., 2016) ha originado que $P$. cinnamomi esté presente en un elevado número de castañares de la Península Ibérica. Otra forma de dispersión de $P$. cinnamomi es a través de zoosporas, que se mueven por sí mismas a cortas distancias en el agua capilar del suelo, o pasivamente arrastradas por el agua gravitacional. Su impacto tanto en pérdidas de individuos como de producción, supone una drástica reducción económica del aprovechamiento que desmotiva al propietario hasta el punto de que éste abandona la gestión o reemplaza la especie.

En el norte de la Península, el impacto de P. cinnamomi se ha visto amortiguado por la utilización de genotipos resistentes obtenidos de cruzar C. sativa con Castanea crenata y Castanea mollissima (Fernández-López, 2011). Sin embargo, en el interior y sur peninsular, la utilización de estos híbridos no es exitosa ni logra reemplazar las masas de castaño anteriormente afectadas por tinta. Se barajan dos hipótesis sobre las dificultades de arraigo del citado material 'resistente' en latitudes sur: (i) no toleran el estrés hídrico estival, o (ii) no resisten las cepas de P. cinnamo- 
mi locales.

Existe un elevado número de trabajos que predicen y modelizan el impacto del cambio climático sobre las enfermedades forestales (Thompson et al., 2014; Woods et al., 2016), pero son necesarios más datos experimentales reales. Por otra parte, también existen estudios sobre el comportamiento del castaño tras la infección con $P$. cinnamomi en distintos ambientes (Gómez-Laranjo et al., 2004; Vanini et al., 2010; Dinis et al., 2011). Sin embargo, hasta el momento no hay información sobre cómo pueden influir condiciones desfavorables previas, que determinan el fenotipo y vigor de la planta, en la susceptibilidad de $C$. sativa a $P$. cinnamomi. Se plantean las hipótesis:

1. Condiciones puntuales de estrés previas a la infección influyen en la mortalidad de $C$. sativa ante $P$. cinnamomi.

2. Escenarios cambiantes generan en la planta mayor susceptibilidad a P. cinnamomi que escenarios estables.

El objetivo del presente trabajo es evaluar la influencia de escenarios cambiantes de estrés hídrico y encharcamiento en la susceptibilidad de C. sativa a P. cinnamomi.

\section{Material y métodos}

Durante el otoño de 2014 se recogieron castañas de Hervás (Cáceres), y en primavera de 2015 se sembraron y germinaron. Durante el período vegetativo de 2015 se utilizaron 576 brinzales, de los cuales aproximadamente un tercio fueron sometidos a condiciones de estrés hídrico $(\mathrm{S})$, un tercio a condiciones de encharcamiento (W) y otro tercio a riego moderado (C) que sirvió como control (Fig. 1).

En la sexta semana del experimento, una parte de los brinzales correspondientes a cada uno de los tratamientos fue inoculada de manera artificial con $P$. cinnamomi. La inoculación se realizó siguiendo el protocolo propuesto por Jung et al. (1996). Dos semanas después de las inoculaciones, las plantas de cada grupo se subdividieron y sometieron a condiciones cruzadas de estrés hídrico $(\mathrm{S})$, encharcamiento $(\mathrm{W})$ y riego moderado (C), de modo que se generaron nueve tratamientos (escenarios cambiantes) y tres controles (escenarios estables) (n-35) (Fig. 1). E1 experimento fue realizado en los invernaderos del Centro Universitario de Plasencia, bajo una malla de sombreo del $50 \%$ de ocultación.

Para constatar la efectividad de los tratamientos se realizaron mediciones periódicas de la humedad del suelo mediante sondas TDR y del estado fisiológico de las plantas (tasa fotosintética (A) y conductancia estomática $\left(\mathrm{g}_{\mathrm{s}}\right)$ ) mediante IRGA (LCi, ADC Bio Scientific Ltd., UK), bajo la malla de sombreo. En estas mediciones se utilizaron 20 plantas por tratamiento.

Se registraron las mortalidades semanalmente (los miércoles) hasta la semana 13 de las inoculaciones. El análisis estadístico de los datos se realizó mediante un análisis ANOVA con el programa STATISTICA V.10. El análisis de supervivencia se realizó a partir del estadístico de Kaplan-Meier, que permite modelizar probabilidades de super- 


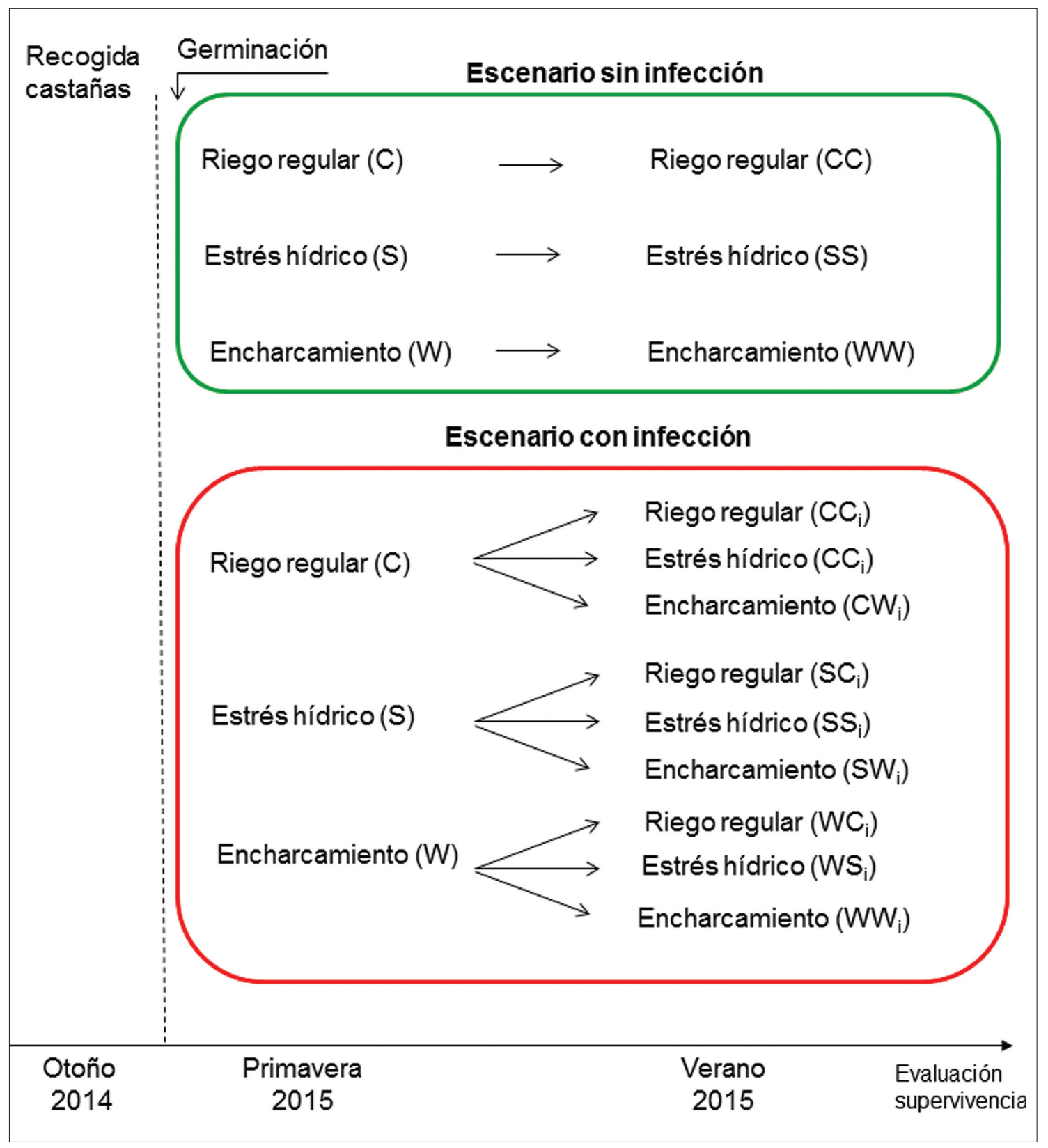

Figura 1. Tratamientos realizados en las plántulas de Castanea sativa bajo los escenarios de infección o no infección con Phytophthora cinnamomi (n-35).

vivencia (Solla et al., 2009). Para comparar diferencias entre los distintos tratamientos se analizaron las supervivencias mediante el test de Wilcoxon-Gehan y las esperanzas de vida de las plantas mediante ANOVAs con el programa STATISTICA V.10.

\section{Resultados y discusión}

En mediciones realizadas a las tres semanas del inicio de los tratamientos, el con- 
tenido de humedad del suelo fue significativamente diferente para los tres grupos de plantas (C, S y W) (Tab. 1). Ello generó menores valores de tasa fotosintética (A) y conductancia estomática $\left(\mathrm{g}_{\mathrm{s}}\right)$ en las plantas estresadas ( SS y WW) frente a las sometidas a riego regular (CC) (Tab. 1), lo que permite constatar que los tratamientos produjeron los efectos esperados en las plantas.

Tabla 1. Valores medios ( \pm error standard; $n=20$ ) de humedad del suelo y parámetros relacionados con la actividad fisiológica de las plántulas evaluadas, obtenidos durante los distintos tratamientos.

\begin{tabular}{lcccc}
\hline & $\mathrm{CC}$ & $\mathrm{SS}$ & $\mathrm{WW}$ & $P$ \\
\hline Humedad $(\% \mathrm{vol})$ & $10.6 \pm 1.1 \mathrm{~b}$ & $3.9 \pm 1.3 \mathrm{c}$ & $21.1 \pm 1.0 \mathrm{a}$ & $<0.01$ \\
$\mathrm{~A}\left(\mu \mathrm{mol} \mathrm{CO} 2 \mathrm{~m}^{-2} \mathrm{~s}^{-1}\right)$ & $4.47 \pm 0.41 \mathrm{a}$ & $2.70 \pm 0.32 \mathrm{~b}$ & $2.86 \pm 0.35 \mathrm{~b}$ & $<0.01$ \\
$\mathrm{gs}_{\mathrm{s}}\left(\mathrm{mmol} \mathrm{H}_{2} \mathrm{O} \mathrm{m}^{-2} \mathrm{~s}^{-1}\right)$ & $75.0 \pm 6.0 \mathrm{a}$ & $31.2 \pm 4.0 \mathrm{~b}$ & $33.3 \pm 4.0 \mathrm{~b}$ & $<0.01$ \\
\hline
\end{tabular}

C, riego regular; S, estrés hídrico; W, encharcamiento; A, tasa fotosintética; gs, conductancia estomática

En la Tab. 2 se muestran los valores de mortalidad para los dieciséis tratamientos evaluados. Empezando por los escenarios sin infección con P. cinnamomi, los resultados correspondientes a los tres tratamientos no alternantes (CC, SS, WW) mostraron diferencias significativas de mortalidad de planta, observándose una mayor tasa de mortalidad en castaños sometidos a encharcamiento permanente (WW) $(P=0.020)$. Estos resultados contrastan con los obtenidos en un experimento similar realizado con Quercus ilex, en el que las plántulas de encina fueron más sensibles a un estrés hídrico prolongado que a un encharcamiento prolongado (Corcobado et al., 2014), lo que indica la poca tolerancia del castaño a la hidromorfía.

Tabla 2. Porcentajes de mortalidad final de plantas de Castanea sativa $(\mathrm{n}>35)$ en función de tres tratamientos (C, S y W) sin y con inoculación de Phytophthora cinnamomi. Valores obtenidos 13 semanas tras las inoculaciones.

\begin{tabular}{|c|c|c|c|c|c|}
\hline $\begin{array}{l}\text { Escenario } \\
\text { sin infección }\end{array}$ & $\begin{array}{l}\text { Tratamiento } \\
\text { Mortalidad (\%) }\end{array}$ & $\begin{array}{c}\mathrm{CC} \\
6.4 \mathrm{a} \mathrm{A}\end{array}$ & $\begin{array}{c}\mathrm{SS} \\
15.2 \mathrm{a} A\end{array}$ & $\begin{array}{c}\text { WW } \\
35.3 \text { b A }\end{array}$ & $\begin{array}{c}P \\
0.020\end{array}$ \\
\hline \multirow[t]{3}{*}{$\begin{array}{l}\text { Escenario } \\
\text { con infección }\end{array}$} & $\begin{array}{l}\text { Tratamiento } \\
\text { Mortalidad (\%) }\end{array}$ & $\begin{array}{c}\mathrm{CC}_{\mathrm{i}} \\
62.4 \mathrm{a} \mathrm{B}\end{array}$ & $\begin{array}{c}\mathrm{CS}_{\mathrm{i}} \\
73.6 \mathrm{a} \mathrm{B}\end{array}$ & $\begin{array}{c}\mathrm{CW}_{\mathrm{i}} \\
100 \mathrm{~b} \mathrm{~B}\end{array}$ & $\begin{array}{c}P \\
0.014\end{array}$ \\
\hline & $\begin{array}{l}\text { Tratamiento } \\
\text { Mortalidad (\%) }\end{array}$ & $\begin{array}{c}\mathrm{SC}_{\mathrm{i}} \\
58.3 \mathrm{a} \mathrm{B}\end{array}$ & $\begin{array}{c}\mathrm{SS}_{\mathrm{i}} \\
80.6 \mathrm{~b} \mathrm{~B}\end{array}$ & $\begin{array}{c}\mathrm{SW}_{\mathrm{i}} \\
82.8 \mathrm{~b} \mathrm{~B}\end{array}$ & $\begin{array}{c}P \\
0.004\end{array}$ \\
\hline & $\begin{array}{l}\text { Tratamiento } \\
\text { Mortalidad (\%) }\end{array}$ & $\begin{array}{c}\mathrm{WCi} \\
63.1 \mathrm{a} \mathrm{B}\end{array}$ & $\begin{array}{c}\mathrm{WS}_{\mathrm{i}} \\
63.4 \mathrm{a} \mathrm{B}\end{array}$ & $\begin{array}{c}\mathrm{WW}_{\mathrm{i}} \\
70.5 \mathrm{a} \mathrm{B}\end{array}$ & $\begin{array}{c}P \\
0.341\end{array}$ \\
\hline
\end{tabular}

$\mathrm{C}$, riego abundante; $\mathrm{S}$, estrés hídrico; $\mathrm{W}$, encharcamiento; $\mathrm{C}_{\mathrm{i}}$, riego abundante tras la infección con $P$. cinnamomi; $\mathrm{S}$, estrés hídrico tras la infección con P. cinnamomi; $\mathrm{W}$, encharcamiento tras la infección con P. cinnamomi. En la misma fila, letras minúsculas distintas indican diferencias significativas al 95\%. En la misma columna, letras mayúsculas distintas indican diferencias significativas al $95 \%$. 
Ante escenarios infectivos la mortalidad de plantas para los mismos tres tratamientos no alternantes fue indiferente al tratamiento $(P=0.279)$, si bien fue más acelerada en WWi que en CCi y SSi (esperanzas de vida de 9.5, 12.6 y 12.0 días, respectivamente). Analizando las esperanzas de vida entre plantas infectadas y no infectadas (factor 1) y entre tratamientos (factor 2), la interacción infección x tratamiento (factor $1 \mathrm{x}$ factor 2$)$ no fue significativa $(P=0.790)$.

Se analizan ahora los resultados teniendo en cuenta variaciones post-inoculación tras un escenario previo común. La mortalidad observada ante un riego favorable (C) previo a la infección con P. cinnamomi fue inferior en CCi y CSi que en $\mathrm{CWi}$ $(P=0.014)$ (Tab. 2), lo que indica un efecto perjudicial exclusivamente del encharcamiento. La mortalidad observada ante un riego escaso (S) previo a la infección con $P$. cinnamomi fue inferior en SCi que en SSi y SWi $(\mathrm{P}=0.004)$ (Tab. 2), lo que indica esta vez un efecto perjudicial del estrés hídrico y el encharcamiento, y un efecto beneficioso de las condiciones favorables de riego. Finalmente, la mortalidad observada ante encharcamiento (W) previo a la infección con $P$. cinnamomi fue indiferente a los escenarios posteriores $(P=0.341)$ (Tab. 2).

Si se analizan los resultados teniendo en cuenta los distintos escenarios previos a la inoculación ante el mismo tratamiento post-inoculación (valores en la misma columna en $T a b .2$ ), en ninguno de los casos se observaron diferencias significativas de mortalidad. En otras palabras, los escenarios previos a la inoculación no influyeron en la mortalidad final de las plantas.

\section{Conclusiones}

Los resultados obtenidos en el presente trabajo constatan la poca tolerancia del castaño a la hidromorfía. Ante escenarios climáticos estables, la mortalidad del castaño por $P$. cinnamomi es indiferente al escenario, pero ocurre antes si el árbol está encharcado. Si las infecciones se producen en escenarios climáticos cambiantes, condiciones favorables al crecimiento del castaño seguidas de encharcamiento generan la máxima mortalidad. Si las infecciones se producen en planta estresada hídricamente, la mortalidad es máxima si las plantas siguen estresadas o si se encharcan, y se reduce si la planta se riega moderadamente. Finalmente, si las infecciones se producen en árboles sometidos a encharcamiento, la mortalidad es independiente al escenario posterior a la inoculación. Eventos desfavorables para la planta previos a infecciones por P. cinnamomi, cortos en duración (tales como el estrés hídrico o el encharcamiento) ni merman ni incrementan la mortalidad final de las plantas. Estos resultados han de ser considerados como preliminares, ya que proceden de plántulas de una savia, y no son directamente trasladables a masas adultas.

\section{Agradecimientos}

Este trabajo ha sido financiado parcialmente a través de los proyectos de inves- 
tigación AGL2013-48017-C2-1-R y AGL2014-53822-C2-1-R, del Ministerio de Economía y Competitividad. M.A. Martín agradece la financiación a la Secretaría General de Ciencia y Tecnología de la Consejería de Economía, Competitividad e Innovación de la Junta de Extremadura (España). Los autores agradecen la colaboración prestada a César Martín (Viveros la Dehesa) por suministrar el material vegetal empleado en el estudio.

\section{Bibliografía}

Alberto, F.J., Aitken, S.N., Alía R., González-Martínez, S.C., Hanninen, H., Kremmer, A., Lefèvre, F., Lenormand, T., Yeaman, S., Whetten, R., Savolainen O., 2013. Potential for evolutionary responses to climate change-evidence from tree populations. Global Change Biol 19, 1645-1661.

Bailey-Serres, J., Colmer, T.D., 2014. Plant tolerance of flooding stress- recent advances. Plant Cell Environ 37, 2211-2215.

Corcobado, T., Cubera, E., Juárez, E., Moreno, G., Solla, A., 2014. Drought events determine performance of Quercus ilex seedlings and increase their susceptibility to Phytophthora cinnamomi. Agric For Meteorol 192, 1-8.

Dinis, L., Peixoto, F., Zhang, C., Martins, L., Costa, R., Gomes-Laranjo, J., 2011. Physiological and biochemical changes in resistant and sensitive chestnut (Castanea) plantlets after inoculation with Phytophthora cinnamomi. Physiol Mol Plant Pathol 75, 146-156.

Fernández-López, J., 2011. Identification of the genealogy of interspecific hybrids between Castanea sativa, Castanea crenata and Castanea mollissima. For Syst 20, 65-80.

Gomes-Laranjo, J., Araujo-Alves, J., Ferreira-Cardoso, Pimentel-Pereira, J.M., Abreu, C.G., J. Torres-Pereira, J., 2004. Effect of chestnut ink disease on photosynthetic performance. J Phytopathology 152, 138-144.

Helfer, S., 2013. Rust fungi and global change. New Phytol 201, 770-780.

Jung, T., Blaschke, H., Neumann, P., 1996. Isolation, identification and pathogenicity of Phytophthora species from declining oak stands. Eur J For Pathol 26, 253-272.

Jung, T., Orlikowski, L., Henricot, B., Abad-Campos, P., Aday, A.G., Aguín Casal, O., Bakonyi, J., Cacciola, S.O., Cech, T., Chavarriaga, D., Corcobado, T., Cravador, A., Decourcelle, T., Denton, G., Diamandis, S., Dogmus-Lehtijärvi, H.T., Franceschini, A., Ginetti, B., Green, S., Glavendekić, M., Hantula, J., Hartmann, G., Herrero, M., Ivic, D., Horta Jung, M., Lilja, A., Keca, N., Kramarets, V., Lyubenova, A., Machado, H., Magnano di San Lio, G., Mansilla Vázquez, P.J., Marçais, B., Matsiakh, I., Milenkovic, I., Moricca, S., Nagy, Z.Á., Nechwatal, J., Olsson, C., Oszako, T., Pane, A., Paplomatas E.J., Pintos Varela, C., Prospero, S., Rial Martínez, C., Rigling, D., Robin, C., Rytkönen, A., Sánchez, M.E., Sanz Ros, A.V., Scanu, B., Schlenzig, A., Schumacher, J., Slavov, S., Solla, A., Sousa, E., Stenlid, J., Talgø, V., Tomic, Z., Tsopelas, P., Vannini, A., Vettraino, A.M., Wenneker, M., Woodward, S., Peréz-Sierra A., 2016. Widespread Phytophthora infestations in European nurseries put forest, semi-natural and horticultural ecosystems at high risk of Phytophthora diseases. For Pathol 46, 134-163.

Mansilla, J.P., Pintos, C., Salinero, M.C., 2000. Plagas y enfermedades del castaño en Galicia. Xunta de Galicia, Consellería de Agricultura, Ganadería e Política Agroalimentaria. 129 pp. 
Martín-García, J., Solla, A., Corcobado, T., Siasou, E., Woodward, S., 2014. Influence of temperature on germination of Quercus ilex in Phytophthora cinnamomi, P. gonapodyides, P. quercina and P. psychrophila infested soils. For Pathol 45, 215-223.

Pautasso, M., Döring, T.F., Garbelotto, M., Pellis, L., Leger, M.J., 2012. Impacts of climate change on plant diseases - opinions and trends. Eur J Plant Pathol 133, 295-313.

Pautasso, M., Holderenrieder, O., 2015. Forest health in a changing world. Microb Ecol 69, 826-842.

Santini, A., Ghelardini, L., De Pace, C., Desprez-Loustau, M.L., Capretti, P., Chandelier,A., Cech, T., Chira, D., Diamandis, S., Gaitniekis, T., Hantula, J., Holdenrieder, O., Jankovsky, L., Jung, T., Jurc, D., Kirisits, T., Kunca, A., Lygis, V., Malecka, M., Mar-cais, B., Schmitz, S., Schumacher, J., Solheim, H., Solla, A., Szabò, I., Tsopelas, P., Vannini, A., Vettraino, A.M., Webber, J., Woodward, S., Stenlid, J., 2013. Biogeographical patterns and determinants of invasion by forest pathogens in Europe. New Phytol 197, 238-250.

Solla, A., Aguín, O., Cubera, E., Sampedro, L., Mansilla, J.P., Zas R., 2009. Survival time analysis of Pinus pinaster inoculated with Armillaria ostoyae: genetic variation and relevance of seed and root traits. Eur J Plant Pathol 130, 477-488.

Stocker, T.F., Qin, D., Plattner, G.K., Tignor, M.B., Allen, S.K., Boschung, J., Nauels, A., Xia, Yu., Bex, V., Midgley, P.M., 2013. Technical Summary. In: Climate Change 2013: The Physical Science Basis. Contribution of Working Group I to the Fifth Assessment Report on the Intergovernmental Panel on Climate Change. Ed. Stocker et al NY, Cambridge University Press.

Sturrock, R.N., Frankel, S.J., Brown, A.V., Hennon, P.E., Kliejunas, J.T., K. J. Lewis, K.J., Worrall, J.J., Woods, A.J., 2011. Climate change and forest diseases. Plant Pathol 60, 133-149.

Thompson, S. E., Levin, S., Rodriguez-Iturbe, I., 2014. Rainfall and temperatures changes have confounding impacts on Phytophthora cinnamomi occurrence risk in the southwestern USA under climate change scenarios. Global Change Biol 20, 1299-1312.

Vanini, A., Natili, G., Anselmi, N., Montaghi, A., Vettraino, A., 2011. Distirbution and gradient analysis of ink disease in chestnut forests. For Path 40, 73-86.

Vieitez, E., Vieitez, M.L., Vieitez, F.J., 1996. El Castaño. Ed. Edilesa, León. 341 pp.

Woods, A.J., Martín-García, J., Bulman, L., Vasconcelos, M.W., Boberg, J., La Porta, N., Peredo, H., Vergara, G., Ahumada, R., Brown, A., Diez, J.J., 2016. Dothistroma needle blight, weather and possible climatic triggers for the disease's recent emergence. Forest Pathol doi: 10.1111/efp.12248

Yáñez-López, R., Torres-Pachecho, I., Guevara-González, R.G., Hernández-Zul, M.I., Quijano-Carranza, J.A., Rico-García, E., 2012. The effect of climate change on plant diseases. Afr J Biotechnol 11, 2417-2428. 